\title{
Ilées \\ actuelles sur la conception des groupes bulbes Exemple de Caderousse
}

\author{
par M. Beslin \\ Compagnie Nationale du Rhône, Lyon
}

Ont participé plus particulièrement à l'élaboration de ce document: MM. BESLIN et LE PLOMB (C.N.R.) - M. RASCALON (SOGREAH)

M. SAURON (Neyrpic) - MM. BOUILLERET, DEBERNADI et MICHAU (Alsthom, Belfort).

\section{INTRODUCTION}

Les trois autres rapports présentés à la séance du Comité Technique pour les groupes bulbes en rivière ayant un caractère général, il a paru utile de centrer la communication sur un cas précis et d'actualité.

Nous avons choisi l'exemple de Caderousse, usine en cours de réalisation, qui comporte à la fois des groupes réglables (distributeur et pales mobiles) et des groupes fixes (distributeur et pales fixes) de très grandes dimensions. Caderousse constitue le dernier gros aménagement du tiers aval du Bas-Rhône, et cet exemple nous permettra, d'une part, de procéder à la synthèse des techniques actuelles des groupes réglables et fixes, d'autre part, d'exposer le point de vue du maître d'œuvre et de l'exploitant, du laboratoire et des constructeurs mécanicien et électricien.

\section{$1^{\text {re }}$ PARTIE}

\section{1. - Zone d'utilisation des groupes bulbes}

Il résulte de l'évolution de la technique des groupes bulbes en rivière, qui vient d'être traitée brillamment dans le rapport précédent, que ce type de machines est maintenant très fiable et qu'il peut être envisagé pour tous les aménagements qui, auparavant, auraient été équipés de turbines Kaplan de basses chutes.

Présentement, avec les groupes bulbes en rivière, it ne semble pas que l'on ait dépassé des hauteurs de chute de l'ordre de 16 à $17 \mathrm{~m}$. Il n'y a pas, en principe, de limite inférieure. Mais, en pratique, pour les grosses machines, elle se situe vers 5 à $6 \mathrm{~m}$. On peut citer, à titre d'exemple, les aménagements de Golfech, sur la Garonne, où la hauteur de chute maximale atteint $17 \mathrm{~m}$, alors que pour le projet de Vaugris, près de Vienne sur le Rhône, la chute est de l'ordre de $6 \mathrm{~m}$. Avec ces machines, on atteint couramment des débits de $400 \mathrm{~m}^{3} / \mathrm{s}$ (aménagements de Vallabrègues, Saint-Vallier et Avignon sur le Rhône). Ces débits sont parfois dépassés: $520 \mathrm{~m}^{3} / \mathrm{s}$ à Saratov en Russie (2 groupes en service); $480 \mathrm{~m}^{3} / \mathrm{s}$ à Rock-Island aux U.S.A. (groupes en projet).

En résumé, on peut dire que les possibilités des gros groupes bulbes sont très comparables à celles des turbines Kaplan de basses chutes en ce qui concerne les débits des machines. Par contre, les hauteurs de chute acceptables pour les machines bulbes ont été jusqu'ici limitées aux environs de $17 \mathrm{~m}$. Au point de vue constructif, on peut certainement dépasser cette valeur, mais nous ne connaissons pas d'étude économique effectuée pour des chutes de l'ordre de $20 \mathrm{~m}$ par exemple. 
De nombreux exposés, présentés à diverses tribunes, ont aussi fait ressortir l'intérêt des groupes bulbes qui permettent, par rapport aux turbines Kaplan, de réaliser des économies substantielles sur les groupes eux-mêmes, sur les auxiliaires et sur le génie civil de l'usine. Aussi, après l'expérience favorable, d'une part de la Rance qui a fait l'objet des deux séances précédentes, d'autre part de Pierre-Bénite, il a été admis que toutes les usines hydroélectriques du Bas-Rhône non encore aménagées seraient équipées avec des groupes bulbes. E.D.F. s'engageait d'ailleurs dans la même voie à partir de Gerstheim et pour les aménagements du Rhin plus en aval.

Pour le Rhône, cette décision exceptait Bourg-les-Valence qui aurait exigé, après l'importante extrapolation de PierreBénite, le lancement d'un nouveau palier pour les machines, sans aucune expérience du précédent. Par contre, cette décision intéressait les six derniers aménagements du BasRhône: soit trois sur le tiers aval (Vallabrègues, Avignon, et Caderousse) et trois sur le tiers amont (Saint-Vallier, Péage-de-Roussillon et Vaugris), pour lesquels les hauteurs de chutes maximales atteignent environ $15 \mathrm{~m}$ à Vallabrègues et au Péage-de-Roussillon, et seulement $6 \mathrm{~m}$ à Vaugris.

Actuellement, outre l'usine de Pierre-Bénite, la plus ancienne équipée en bulbes, les usines de Vallabrègues et de Gervans (aménagement de Saint-Vallier) sont en exploitation; l'aménagement d'Avignon doit être mis en service au début de 1973 et les travaux de l'aménagement de Caderousse sont en cours pour une mise en service en 1975. Les travaux du Péage-de-Roussillon et de Vaugris doivent être engagés pour aboutir à un aménagement hydroélectrique complet du Bas-Rhône et à une voie navigable continue de Lyon à la mer vers 1978.

En résumé, grâce à la technique récente des groupes bulbes, sept usines hydroélectriques du Bas-Rhône sur douze seront équipées avec ce type de machines.

\section{2. - Dimensionnement des machines}

\subsection{Caractéristiques générales des usines bulbes en} service ou en construction sur le Rhône.

Les aménagements de Vallabrègues, Saint-Vallier et Avignon sont prévus pour un débit d'armement d'environ:

$$
\begin{aligned}
& -2400 \mathrm{~m}^{3} / \mathrm{s} \text { à Vallabrègues; } \\
& -1600 \mathrm{~m}^{3} / \mathrm{s} \text { à Saint-Vallier; } \\
& -2400 \mathrm{~m}^{3} / \mathrm{s} \text { à Avignon. }
\end{aligned}
$$

$\mathrm{Si}$, pour ces trois aménagements, on compare les « courbes de débits unitaires classés» obtenus en divisant les débits classés du Rhône par le nombre de groupes ( 6 à Vallabrègues, 4 à Saint-Vallier et 6 à Avignon), on constate que ces trois courbes sont presque superposées. Quant aux courbes de «hauteurs de chute classées», elles sont également très voisines, l'écart atteignant environ $0,60 \mathrm{~m}$ dans la zone du débit d'équipement.

Dans ces conditions particulières, l'optimisation des dimensions, en vue d'obtenir l'enrichissement maximal, conduit, pour toutes ces usines, aux mêmes résultats, ce qui a permis d'équiper ces trois aménagements avec des groupes bulbes (turbines et alternateurs) présentant des caractéristiques identiques, à savoir:

- débit unitaire maximal .......... $400 \mathrm{~m}^{3} / \mathrm{s}$

— diamètre de roue ........................ $6,25 \mathrm{~m}$

- vitesse de rotation ............ 93,75 $\mathrm{tr} / \mathrm{mn}$

— puissance .................... $35 \mathrm{MW}$

On perçoit tout de suite l'intérêt que peut présenter, pour le maître-d'œuvre et l'exploitant, l'exécution de machines identiques.

Toutes ces machines sont du type bulbe amont à double réglage et comportent, comme celles de la Rance et de Pierre-Bénite, des distributeurs et pales mobiles.

Ce type d'équipement constitue un palier d'économie important par rapport aux solutions Kaplan verticales classiques. On sait, cependant, que l'allègement des machines ne se traduit pas par un allègement proportionnel des prix, du fait, notamment, que certains éléments sont de réalisation plus difficile que pour les machines vericales. Il en est notamment ainsi du distributeur sphérique, et les maîtres d'œuvre, tant E.D.F. que C.N.R., ont, très tôt, cherché la possibilité de réaliser des groupes à distributeur fixe $\left({ }^{a}\right)$. Malheureusement, pour tous les sites courants en rivière, il apparaissait que l'économie ainsi réalisée était à peu près intégralement détruite par l'abaissement de la production, abaissement qui résulte de l'adaptation moins parfaite de ces machines à des variations importantes de chutes et de débits. Il apparaissait qu'une telle solution aurait, par contre, toutes les chances d'être économiquement intéressante dans un site semblable à ceux du cours inférieur du Saint-Laurent, où débits et chutes ne sont que très peu variables.

La branche est de l'aménagement d'Avignon devait fournir le site favorable à ce changement de technique.

\section{CAS PARTICULIER de L'AMÉNAGEmENT D'AVIGNoN.}

Pour des raisons géographiques particulières, l'aménagement d'Avignon comporte deux usines, fonctionnant en parallèle, sur deux bras du fleuve.

De longues études, menées en accord avec les services d'Electricité de France, ont conduit à adopter l'équipement «mixte » suivant :

- la première usine, dite usine d'Avignon, située sur le bras ouest du Rhône, est équipée pour un débit de $1600 \mathrm{~m}^{3} / \mathrm{s}$; elle comporte quatre groupes mobiles à double réglage, identiques à ceux de Saint-Vallier;

- la seconde, dite usine de Sauveterre, implantée sur le bras est, est équipée pour un débit de $800 \mathrm{~m}^{3} / \mathrm{s}$; elle comporte deux groupes à débit fixe, sans réglage.

Ainsi le débit global d'équipement de la chute d'Avignon à $2400 \mathrm{~m}^{3} / \mathrm{s}$ résulte de la similitude de débit et de chute entre l'aménagement d'Avignon et l'aménagement de Vallabrègues.

La répartition en deux usines résulte de la condition, fixée par le Cahier des Charges de la chute, qui impose de

(a) Le lecteur pourra, s'il le désire, se rapporter à larticle de M. Cazenave, Directeur des Services Electromécaniques à la Compagnie Nationale du Rhône, sur l'usine de Pierre-Bénite, article paru dans la revue Travaix (n" 392 de novembre 1967). 
maintenir, en permanence, dans la branche est (ou bras d'Avignon), un débit minimum de $400 \mathrm{~m}^{3} / \mathrm{s}$.

Du fait de cette condition, l'examen des courbes de débits et de chutes à l'usine de Sauveterre montre, pendant 270 jours par an, un débit constant de $400 \mathrm{~m}^{3} / \mathrm{s}$, la chute étant également pratiquement constante: la variation, durant cette même période, est inférieure à $0,50 \mathrm{~m}$.

Les caractéristiques de la chute d'Avignon: débits, hauteurs de chute et puissances classées sont concrétisées dans la figure 1.

La Production Hydraulique d'E.D.F., mise au courant de ces données d'exploitation particulières, a non seulement accepté notre proposition de supprimer les distributeurs, mais s'est même déclarée prête à renoncer à tout réglage sur Sauveterre (au prix d'une petite complication sur la répartition entre les deux bras), s'il pouvait en résulter des machines plus simples.

De cet accord, est partie l'étude de groupes de $400 \mathrm{~m}^{3} / \mathrm{s}$ chacun, à distributeur et pales fixes. Ces deux groupes, qui fonctionnent par tout ou rien (le réglage des débits étant assuré par l'usine d'Avignon), permettent des économies substantielles d'investissement, sans altération importante des performances. Pour ces groupes, le diamètre de roue a été porté en définitive à $6,90 \mathrm{~m}$, cet accroissement des dimensions, qui résulte d'un calcul d'optimisation économique, étant rendu possible par l'allègement du palier turbine à la suite de la suppression du mécanisme de commande des pales. On sait en effet que, dans les groupes bulbes Kaplan, c'est la charge sur le palier turbine qui conditionne pratiquement les dimensions de la machine.

Ainsi, verront le jour à l'usine de Sauveterre, les groupes bulbes simplifiés ou groupes «fixes» $\left({ }^{b}\right)$ qui constituent une «troisième génération 》de groupes bulbes, la première étant celle des microcentrales, la seconde celle des gros groupes réglables.

Pour mesurer la portée de ce nouveau palier technique, on peut dire que, tous comptes faits, le supplément d'enrichissement $\left(^{\circ}\right)$, qui résulte de l'adoption de cette nouvelle technique, représente entre 60 et $80 \%$ du supplément d'enrichissement qui avait été constaté lors du passage de la technique verticale à celle des bulbes réglables.

\section{3. - Projet d'usine de Caderousse}

Les courbes des débits classés à Avignon et à Caderousse sont identiques, le Rhône ne recevant dans l'intervalle aucun affluent important.

Les courbes des hauteurs de chutes classées à Avignon et Caderousse sont également très proches l'une de l'autre, la chute à Caderousse étant cependant inférieure d'environ $0,40 \mathrm{~m}$ à celle d'Avignon, avec une hauteur de chute moyenne disponible de $9,15 \mathrm{~m}$ environ. Pour toutes cer raisons, l'équipement de Caderousse devait normalement êtrê identique, quant au débit d'armement, à celui d'Avignon.

(b) Expression elliptique qui rappelle que le distributeur fixe et que l'inclinaison des pales est également fixée.

(c) Ou, si l'on préfère, l'économie nette, compte tenu des écarts de puissance et de production.

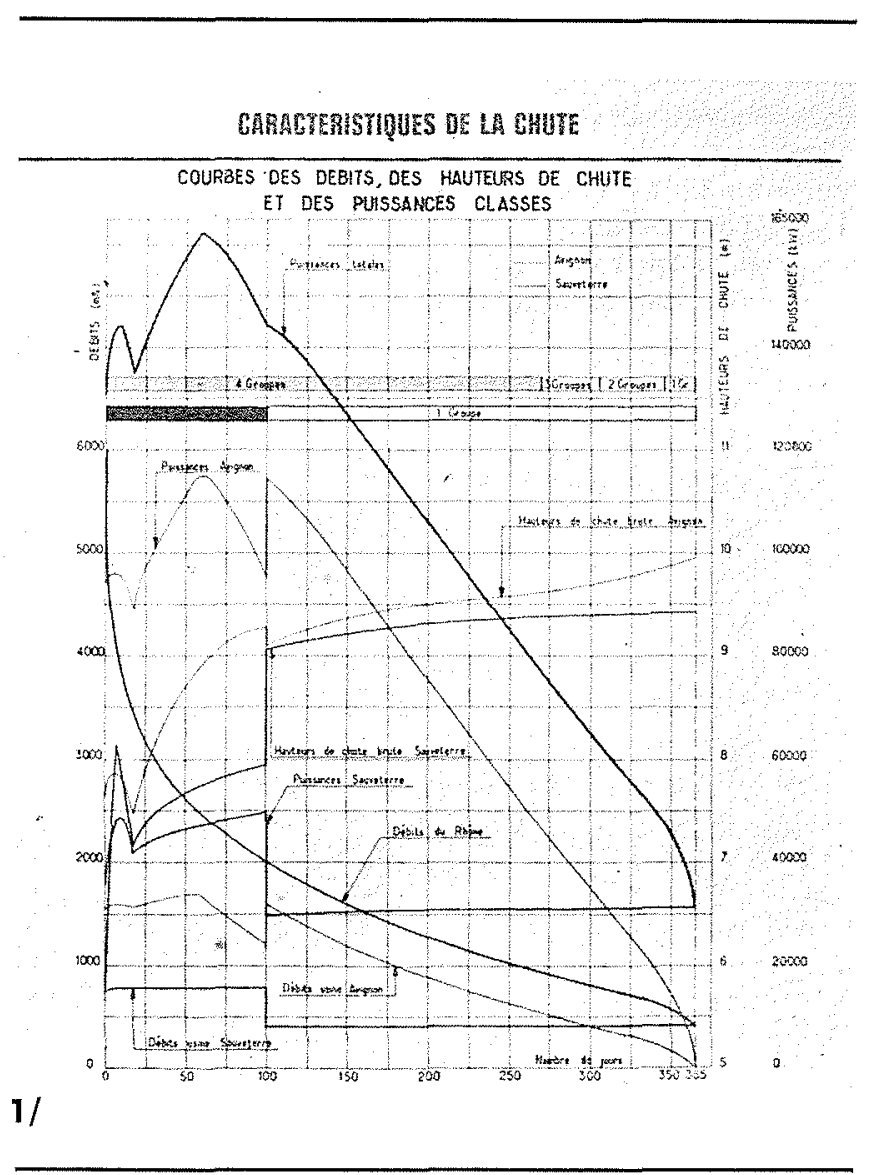

\subsection{Détermination du débit d'équipement.}

L'équipement optimal de Caderousse doit comporter, dans l'hypothèse de machines présentant un débit unitaire de l'ordre de $400 \mathrm{~m}^{3} / \mathrm{s}$, soit cinq groupes minimum, soit six groupes. Nous avons donc comparé, dans la gamme des débits d'équipement possibles entre 2000 et $2400 \mathrm{~m}^{3} / \mathrm{s}$, les solutions à cinq ou six groupes.

Pour un même débit d'équipement, chacune de ces solutions conduisait à des enrichissements très voisins. Ce résultat donnait un très léger avantage à la solution cinq groupes.

Toutefois, avec cette hypothèse, il ne nous paraissait pas prudent de procéder à une nouvelle extrapolation de la taille des machines, dont le diamètre de roue devait être porté à $6,70 \mathrm{~m}$ environ, pour absorber le débit global de $2400 \mathrm{~m}^{3} / \mathrm{s}$ (soit $480 \mathrm{~m}^{3} / \mathrm{s}$ par machine). Pour rester dans la gamme des solutions éprouvées, nous avons admis de ne pas dépasser un diamètre de $6,35 \mathrm{~m}$, ce qui limitait le débit d'équipement à $2100 \mathrm{~m}^{3} / \mathrm{s}$ environ.

Dans le cas d'une usine à six groupes, l'enrichissement maximal a lieu pour un débit d'équipement de $2400 \mathrm{~m}^{3} / \mathrm{s}$ et cet enrichissement dépasse encore de plusieurs millions de francs l'enrichissement correspondant de l'usine à cinq groupes équipée, compte tenu du maximum de diamètre fixé ci-dessus, pour $2100 \mathrm{~m}^{3} / \mathrm{s}$.

Le débit a donc en définitive été fixé à $2400 \mathrm{~m}^{3} / \mathrm{s}$, et réparti sur six machines.

\subsection{Possibilités d'exploitation de l'usine de Caderousse.}

Le débit d'armement étant ainsi arrêté, il était tentant d'examiner, pour cette usine, la possibilité de réaliser un 
équipement: soit entièrement avec des groupes à débit fixe, soit avec les deux types de machines, mentionnés ci-avant, pour les usines d'Avignon et de Sauveterre.

La première hypothèse a été abandonnée, car elle conduisait, pour l'usine de Caderousse, soit à une exploitation à déversement permanent avec de grosses pertes d'énergie, soit à une exploitation à puissances variables, incompatible notamment avec les besoins du réseau.

L'exploitation à déversement permanent aurait conduit à déverser, en moyenne, $200 \mathrm{~m}^{3} / \mathrm{s}$ environ durant 270 jours par an, où le débit naturel est inférieur au débit d'équipement. Il s'en suit une perte d'énergie annuelle d'environ $100 \mathrm{GWh}$ et une perte en capital nettement supérieure aux économies que l'on pouvait escompter sur l'équipement.

Avec l'exploitation à niveau variable, le débit turbiné, réalisé avec les machines à débit fixe, est compris entre le débit immédiatement inférieur au débit naturel, et le débit réalisable immédiatement supérieur.

Ce mode d'exploitation, qui aboutit à une exploitation permanente en éclusées, fait perdre, du point de vue énergétique seulement, $15 \mathrm{GWh} / \mathrm{an}$, mais l'énergie produite ne correspond pas exactement aux besoins quotidiens du Mouvement d'Energie. En définitive, cette exploitation à niveau variable était grevée, au départ, de beaucoup d'inconvénients et d'inconnues (démarrages fréquents des machines, qualité de l'énergie produite, perturbations des possibilités d'éclusées de la chaîne d'usines).

Nous avons donc évolué naturellement vers une usine comportant un équipement «mixte», permettant de bénéficier des avantages des groupes réglables (à pales et distributeur mobiles) pour le réglage du débit, et de ceux des groupes fixes pour l'économie. Les groupes fixes assureraient la base du débit de l'usine, alors que les groupes réglables seraient chargés d'effectuer les modulations et variations périodiques.

L'équipement mixte de l'usine étant ainsi adopté, la mise au point du projet a été poursuivie avec le souci d'aboutir à une usine homogène, quant aux fondations des groupes, aux tracés hydrauliques, aux circulations et à l'aspect général.

\section{4. - Projet d'usine avec équipement mixte}

\subsection{Dispositions générales.}

L'équipement définitif de l'usine de Caderousse est donc fixé comme suit, avec des machines dont les caractéristiques sont identiques à celles des usines d'Avignon et Sauveterre, à savoir :

- 2 groupes réglables, diamètre de roue $6,25 \mathrm{~m}$;

- 4 groupes fixes, diamètre de roue $6,90 \mathrm{~m}$.

La vitesse de rotation est fixée uniformément à $93,75 \mathrm{tr} / \mathrm{mn}$ et la puissance active des alternateurs atteint $31,5 \mathrm{MW}$.

L'usine de Caderousse, qui évacue son énergie vers le poste de l'Ardoise, situé à proximité en R.D., par l'intermédiaire de deux lignes distinctes afin de diminuer les risques dus aux ondes de déclenchement, est divisée en deux demi-usines.
Pour chaque demi-usine, on avait le choix pour l'équipement entre deux solutions:

- soit un groupe réglable et deux groupes fixes;

- soit deux groupes réglables et un groupe fixe.

Dans l'hypothèse où un groupe réglable de la centrale serait en entretien, chacune des solutions ci-dessus permet une exploitation au fil de l'eau et en éclusées satisfaisante. Même dans le cas où l'on ne disposerait à la centrale que de deux groupes réglables, la modulation du débit peut encore être assurée avec le seul groupe réglable de l'autre demi-usine.

Les problèmes techniques étant ainsi posés, le choix entre l'une ou l'autre des deux solutions envisagées ci-dessus résulte de considérations économiques.

Nous avons ainsi comparé, par rapport à une usine à six groupes réglables, les enrichissements d'une usine équipée de quatre groupes réglables avec deux groupes fixes, ou de deux groupes réglables avec quatre groupes fixes.

L'enrichissement procuré par la seconde solution, compte tenu de tout (notamment la vanne amont) $\left({ }^{d}\right)$ est sensiblement le double de celui donné par la première solution et atteint environ $16 \mathrm{MF}$ en valeur actuelle. Ces résultats montrent que l'équipement optimal doit comporter deux groupes réglables et quatre groupes fixes.

En premier lieu, l'usine de Caderousse est construite à ciel ouvert, de conception dite out door, au même titre que les usines de Vallabrègues, Avignon et Sauveterre. Les groupes réglables qui, en principe, sont toujours en service, sont disposés au centre de chaque demi-usine et bénéficient ainsi d'une alimentation meilleure que s'ils étaient disposés sur les rives. En outre, il est intéressant, lors de la mise en service de l'usine, de disposer assez vite d'un groupe réglable, capable d'assurer le réglage et le transfert du débit du canal d'amenée vers l'aval.

Les groupes fixes conduisent à adopter une entrée en chambre d'eau ouverte, qui joue le rôle de cheminée d'équilibre, et qui permet la coupure d'extrême sécurité avec la vanne banalisée amont évoquée ci-avant. La disposition en chambre d'eau ouverte a été étendue aux groupes réglables, ce qui entraîne, pour ces groupes, la suppression de la galerie traditionnelle amont aménagée à l'intérieur de l'ogive. En conséquence, on a dû modifier, comme on l'indique ci-après, la structure du groupe en vue de reprendre tous les efforts transmis aux maçonneries.

Les entrées d'eau des groupes fixes et réglables sont pratiquement identiques. C'est ainsi que les grilles et les rainures à batardeau amont, de tous les groupes, sont impérativement alignées pour l'organisation du dégrillage et pour la circulation des portiques nécessaires au batardement et aux opérations de montage et de démontage des matériels.

Les rainures du batardeau amont sont organisées pour recevoir le batardeau amont d'exploitation, ainsi que la vanne de sécurité amont banalisée.

\subsection{Structures des machines.}

Nous ne nous étendrons pas sur la description des machines (turbines alternateurs), qui sera développée par le constructeur turbinier et par le constructeur électricien dans la

(a) Le rôle de la vanne amont sera précisé plus loin. 
troisième partie. Nous ne donnons, ci-après, qu'une description très générale de l'ensemble turbine-alternateur.

Pour les groupes réglables, on a donc remplacé la galerie traditionnelle par un avant distributeur, qui reprend la majeure partie des efforts et qui est noyé dans le béton du mur aval de la chambre d'eau.

Pour les groupes fixes, le distributeur, qui remplit le même office que l'avant distributeur des groupes réglables, est constitué par un anneau complet très rigide, fortement entretoisé, également mis en place dans le mur aval de la chambre d'eau.

Pour les deux types de groupes, on trouve de l'amont à laval:

- L'ogive hémisphérique, qui assure la continuité du profil hydraulique côté alternateur.

- Le croisillon, en une seule pièce, qui est fixé entre l'ogive et la bride amont de l'alternateur.

Le croisillon du groupe réglable supporte à la fois le palier alternateur et la butée, alors que le croisillon du groupe fixe supporte seulement le palier alternateur, la butée étant reportée au niveau de l'accouplement des arbres turbine et alternateur.

le croisillon est boulonné sur deux pieds verticaux ancrés dans le béton du radier de la chambre d'eau.

- Lalternateur, fixé entre le croisillon ci-dessus et le distributeur (ou l'avant distributeur, suivant qu'il s'agit d'un groupe fixe ou réglable).

L'accès, à l'intérieur de chaque machine, s'effectue, depuis la galerie de drainage inférieure, par l'un des pieds supports mentionnés ci-avant.

Cette galerie de drainage devient, ainsi, la galerie d'exploitation et de circulation pour toute l'usine.

\subsection{Calage des machines.}

Toujours pour des raisons d'uniformité, le calage de toutes les machines, dont les pales sont en acier à $13 \%$ de chrome, a été fixé uniformément à la cote $(11,00)$. Cette cote est définie pour les groupes réglables et réserva une marge d'au moins $10 \%$, dans les cas les plus défavorables, entre le $\sigma$ standard défini sur modèle réduit et le $\sigma$ d'installation.

En application de règles semblables, les groupes fixes, du fait que leur débit spécifique est plus faible, auraient pu être calés environ $2,00 \mathrm{~m}$ ou $2,50 \mathrm{~m}$ plus haut, mais on a voulu éviter les faux niveaux à l'usine, ceux-ci étant peu compatibles avec une exploitation dont la circulation s'effectue, comme nous l'avons déjà dit, à partir de la galerie la plus basse de l'usine. Les groupes fixes bénéficient ainsi d'une marge de sécurité supplémentaire à l'égard de la cavitation.

\subsection{Vannes aval.}

Les vannes aval des groupes réglables et fixes ont un triple rôle et doivent assurer:

- la protection du groupe en cas d'emballement;

- le rétablissement du débit, en cas de disjonction, grâce à la marche en déchargeur, qui permet de faire transiter vers l'aval environ 70 à $75 \%$ du débit de pleine charge;
- le batardement aval du groupe.

Au surplus, pour les groupes fixes qui ne comportent aucun organe de réglage de débit, donc de la puissance, les démarrages et arrêts de machines, sont encore assurés avec les vannes aval.

Au démarrage, le groupe est couplé en asynchrone sur le réseau, après avoir été amené, par ouverture progressive de la vanne aval, à une vitesse très proche de la vitesse synchrone.

L'arrêt est obtenu par descente de la vanne aval et disjonction au voisinage de la puissance nulle.

Les vannes aval des six groupes ont été toutes alignées et implantées à une distance de $18,35 \mathrm{~m}\left(^{\circ}\right)$ de l'axe des groupes réglables (soit $2,93 d$ environ). Cette position correspond sensiblement à la position optimale obtenue en tenant compte, d'une part des investissements en matériel et en génie civil, d'autre part des pertes d'énergie imputables à la présence de la rainure de vanne.

Toutes les vannes ont une section identique, cette condition ayant été rendue possible par la réduction de la conicité de l'aspirateur des groupes fixes (ramenée à $5^{\circ} 30$ ), alors que les aspirateurs des groupes réglables présentent la conicité habituelle de $6^{\circ} 30$.

\subsection{Vanne de sécurité amont.}

La vanne de sécurité amont, utilisée à titre d'ultime sécurité, interviendrait dans le cas où la vanne de sécurité aval d'un groupe fixe à l'emballement, ne pourrait être descendue. La vanne amont, banalisée pour toute l'usine, est composée de deux éléments solidarisés à l'aide de bretelles qui permettent, normalement, un écartement de ces éléments. La vanne est descendue à l'aide des deux portiques de l'usine, en maintenant une lumière entre les deux éléments, permettant ainsi une meilleure alimentation du groupe durant la coupure. La persienne se referme lorsque, l'élément inférieur reposant sur le seuil, l'élément supérieur poursuit seul sa descente, assurant la fermeture totale du pertuis. La coupure se termine donc sensiblement dans l'axe de la machine.

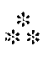

Les dispositions générales de l'usine de Caderousse, auxquelles il est fait allusion ci-avant, sont concrétisées par. les figures suivantes:

- Coupe d'un groupe réglable (fig. 2).

- Coupe d'un groupe fixe (fig. 3).

- Vue en plan de l'usine (fig. 4).

Les coupes d'usines antérieures permettent la comparaison avec l'usine de Caderousse:

- Usine de Vallabrègues (fig. 5).

- Usine de Saint-Vallier (fig. 6).

- Usine d'Avignon (fig. 7).

- Usine de Sauveterre (fig. 8).

(c) Distance déjà retenue pour Saint-Vallier et Avignon et qui permet d'obtenir les mêmes sections de vannes et des bouchures normalisées. 


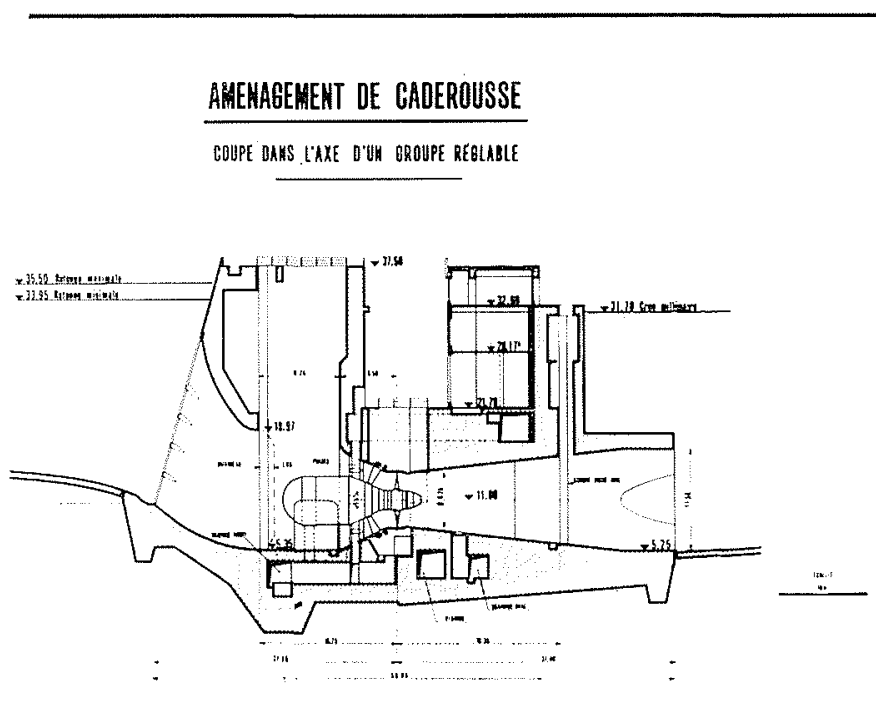

2/

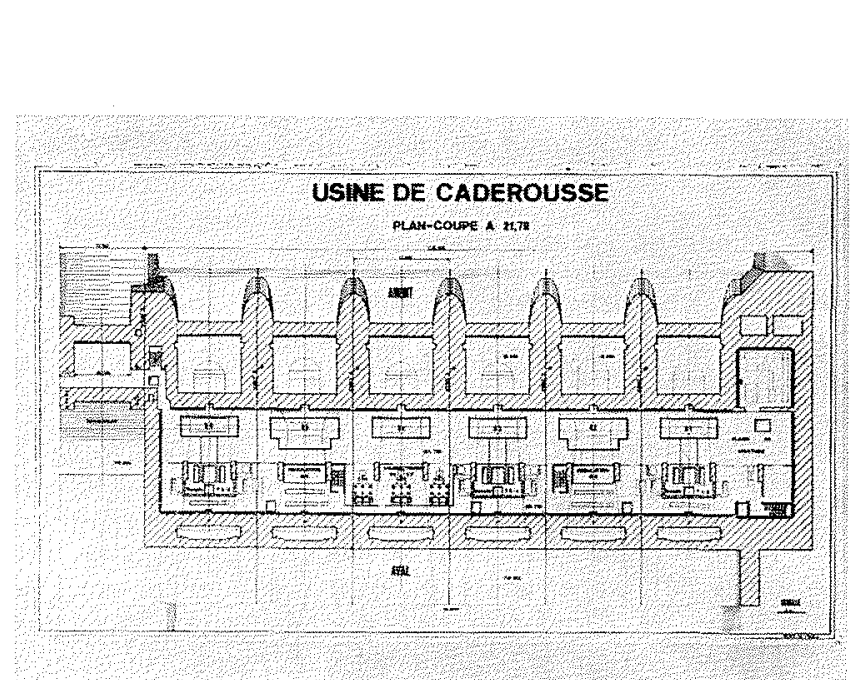

$4 /$

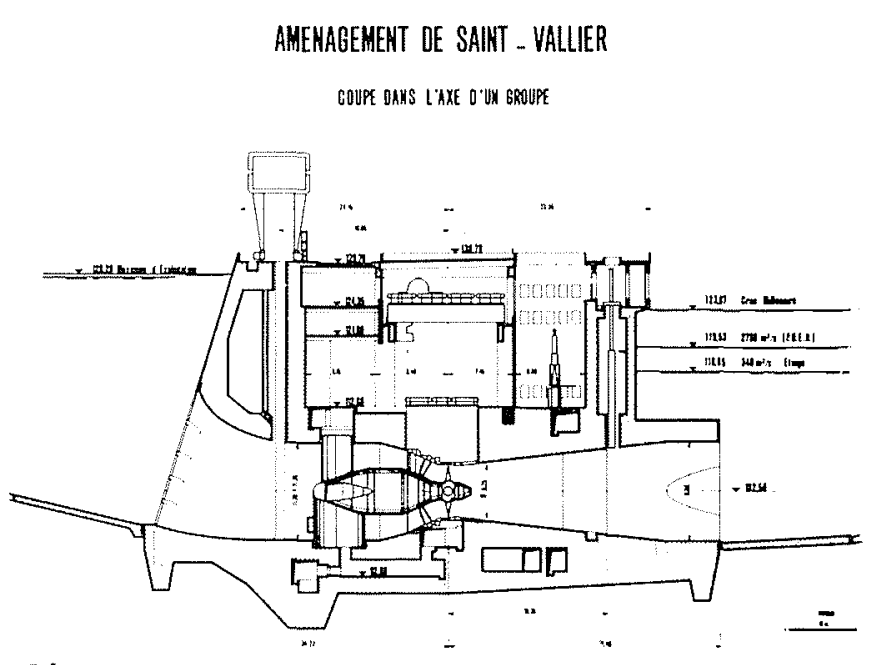

$6 /$
AMEEAGEAHEHT DE CADEROUSSE

COBPE OAHS LAXE D'UK GROUPE FIYE

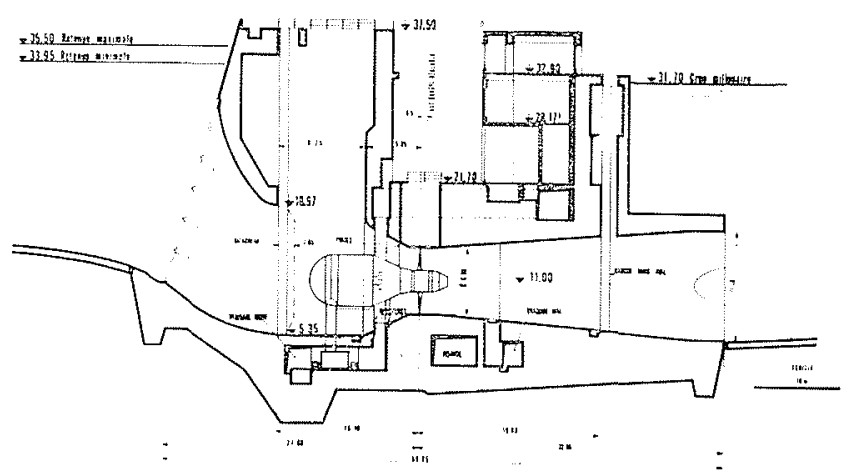

$3 /$
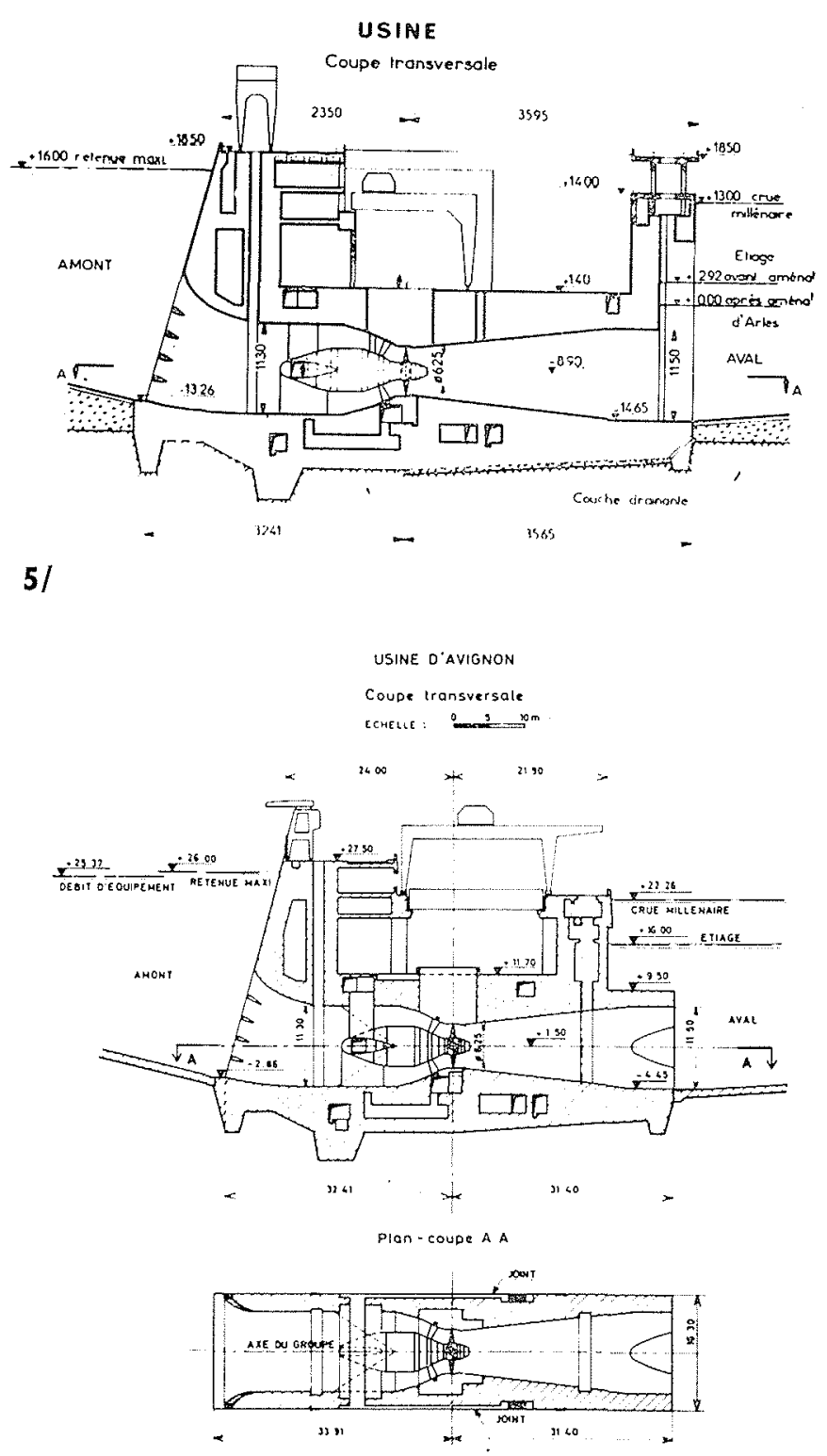

71 


\section{5. - Performances \\ des groupes simplifiés : Rendement, Marche en déchargeur}

Les performances des groupes fixes, quant au rendement et à la marche en déchargeur, peuvent être considérées comme étant sensiblement équivalentes à celles des groupes réglables.

\subsection{Rendement.}

Par construction, la forme du distributeur et l'inclinaison des pales sont définies pour la chute et le débit dans la zone de l'étiage, et ces caractéristiques varient assez peu durant environ les trois-quarts de l'année. Les groupes fixes fonctionnent à $q_{11}$ et $n_{11}$ relativement constants durant toute cette période où le rendement diminue lentement. Mais il chute assez rapidement (de 3 à 4 points) lorsque l'on atteint les fonctionnements à forts débits et basses chutes.

Dans le cas de Caderousse, on note, pour les groupes fixes, des rendements modèles garantis pondérés qui ne sont guère inférieurs de plus de $2 \%$ aux rendements pondérés obtenus pour les groupes mobiles.

C'est ainsi que la perte globale d'énergie, entre la solution comportant uniquement des groupes réglables et la solution mixte retenue en définitive pour Caderousse, n'atteint pas $2 \%$. Cette perte de production est largement justifiée par les économies d'investissement comme on l'a vu ci-avant.

\subsection{Marche en déchargeur.}

Le débit restitué au bief aval, par la marche en déchargeur, est le même pour les groupes fixes que pour les groupes réglables, soit pour le cas qui nous occupe au moins $280 \mathrm{~m} \% \mathrm{~s}$, soit $70 \%$ du débit de pleine charge.

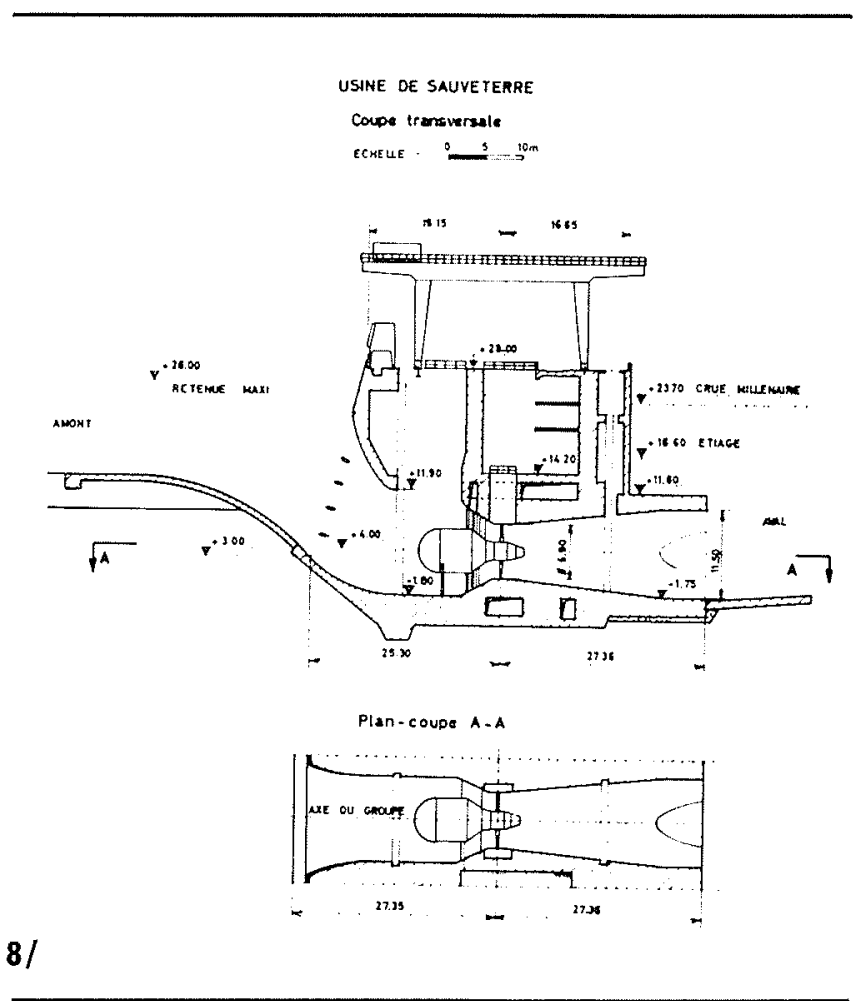

Par suite de l'absence de distributeur mobile, on note, au début de la séquence de fonctionnement en déchargeur des groupes fixes, une survitesse et un surdébit plus importants par rapport aux constatations faites pour les groupes réglables. Le bief amont est donc l'objet, d'abord d'une onde négative, d'amplitude très modérée grâce à l'intervention rapide de la vanne aval, puis d'une onde positive.

\section{$2^{\mathrm{e}}$ PARTIE}

\section{Problèmes concernant le tracé hydraulique des groupes bulbes}

L'équipement des basses chutes, avec des groupes bulbes de dimensions raisonnables et de forte puissance unitaire, impose la réalisation de turbines de très grands débits et vitesse spécifiques. L'implantation possible de l'alternateur dans un bulbe immergé mène, tout naturellement, à un groupe axial, implanté dans un conduit horizontal, afin d'aboutir au trajet le plus direct possible des filets d'eau.

Nous examinons, de l'amont vers l'aval, les problèmes posés par un tel tracé qui intéresse à la fois les groupes mobiles et les groupes fixes.

\section{Radier amont de l'usine.}

La forme du radier, placé en amont de l'usine, doit faire l'objet d'une étude sur modèle réduit pour obtenir une alimentation aussi uniforme que possible des machines.

Il faut éviter les décollements des filets d'eau dans les zones de changement de pentes du radier et des vortex en surface. En outre, les fouilles amont sont conditionnées par l'altitude du radier de la prise d'eau.

\section{Entonnement.}

Il doit assurer une alimentation homogène, non seulement du bulbe lui-même, mais aussi de toute la surface des grilles d'entrée, car, si cette dernière condition n'est pas satisfaite, les pertes dues au passage de la grille peuvent être relativement très importantes par rapport à la chute. Il y a un net avantage à rechercher des vitesses aussi peu inclinées que possible par rapport au plan de la grille, en s'efforçant de ne pas dépasser une valeur de l'ordre de 15 à 20"; au-delà de ces valeurs, les pertes de charge deviennent importantes et dépassent $0,2 \mathrm{~V}^{2} / 2 \mathrm{~g}$ ( $\mathrm{V}$ : vitesse d'approche).

L'implantation du batardeau amont, placée derrière les grilles, impose une section rectangulaire, et de dimensions raisonnables.

Une bonne approche des pertes de charge dans le conduit et imputables aux grilles, aux rainures du batardeau amont et de la vanne aval et à la restitution en aval peut être donnée par la formule $=0,005 q_{11}^{2}\left(q_{11}\right.$ : débit spécifique). 


\section{Conduit du bulbe.}

Le diamètre du bulbe dépend de l'encombrement de l'alternateur. On note des rapports diamètre de roue/diamètre alternateur qui varient couramment de 0,80 à 0,90 .

L'entrée dans le distributeur étant obligatoirement de section circulaire, cela nécessite, sur la longueur du bulbe, un raccordement carré-cercle d'une convergence régulière, malgré la présence des obstacles nécessaires à la rigidité et à la tenue du bulbe, tels que : ogive avec ses bras horizontaux ou ogive avec ses pieds verticaux scellés dans le radier.

\section{Ensemble avant distributeur et distributeur.}

Dans le cas des groupes réglables, l'avant distributeur est généralement constitué d'ailettes radiales: son rôle directionnel est faible et il assure surtout la stabilité mécanique du bulbe. Le distributeur mobile est conique pour s'inscrire au mieux entre le bulbe et la roue : il comporte en général vingt-quatre directrices réglables, relativement courtes.

Un certain nombre de groupes d'une centrale peuvent être privés d'organes de réglage, l'avant distributeur n'existe plus et l'ensemble distributeur est alors fixe et comporte un tracé «maillé» établi pour le fonctionnement le plus courant de la machine. Dans ce cas, les directrices sont plus importantes, et assurent à la fois la stabilité mécanique de la machine et un rôle directionnel des filets liquides.

\section{Roue.}

Le tracé des pales dépend de l'écoulement délivré par le distributeur et des caractéristiques du moyeu et de la ceinture de roue, généralement cylindro-sphérique. Pour les hauteurs de chute du Bas-Rhône et les débits unitaires des machines, le nombre de pales est couramment de quatre.

Les conditions d'écoulement en aval du distributeur, l'encombrement de la commande des pales pour les groupes mobiles et la fixation des pales des groupes fixes conditionnent le dimensionnement du moyeu. Pour les groupes de Caderousse, le rapport de moyeu, entre groupes fixes et groupes réglables, atteint environ 0,84 .

\section{Aspirateur.}

C'est, en général, un cône droit dont l'angle est adapté à l'écoulement délivré par la roue: le rendement d'un tel aspirateur est nettement supérieur à celui d'un aspirateur coudé.

A Caderousse, les aspirateurs des groupes réglables ont une conicité de $6 \% 30$, alors que celle des groupes fixes est d'environ $5^{\circ} 30$. Cet angle réduit est imposé, d'une part, par l'implantation de vannes aval de dimensions uniformes, et d'autre part, par la présence d'une ceinture de roue cylindrique, sans col, qui tolère mal, dans l'aspirateur, une divergence aussi importante que celle admise en aval des ceintures sphériques des groupes réglables.

La forme et les dimensions de la section de sortie ont été étudiées pour minimiser l'énergie cinétique subsistant à ce niveau.

Chaque groupe bulbe est muni d'une vanne aval, nécessaire pour le fonctionnement du groupe en déchargeur, ainsi que pour la protection à l'emballement de la machine.

\section{Radier aval.}

Il peut concourir, par un tracé judicieux, à la récupération d'une part importante de l'énergie cinétique libérée par l'aspirateur $\left({ }^{(}\right)$.

\section{$3^{c}$ PARTIE}

\section{1. - Evolution de la conception mécanique des groupes bulbes}

\subsection{Généralités.}

Depuis la mise au point des groupes bulbes de la Rance et de la centrale de Pierre-Bénite, les recherches conjuguées des maîtres d'œuvre (Electricité de France et C.N.R.) et des constructeurs ont été poursuivies dans deux directions:

a) Amélioration de la conception des groupes réglables (pales et directrices mobiles).

Parmi les principaux résultats obtenus, on peut citer, en prenant, pour base la conception des groupes de PierreBénite et dans l'ordre :

Beaucaire, Gervans et Avignon:

- Suppression de la réfrigération de l'alternateur en paroi dans l'ogive et les bras d'accès, et report de cette réfri- gération en paroi de la carcasse des alternateurs (voir 5.2.1).

- Remplacement de la solution classique, anneau de scellement amont scellé - avant-distributeur démontable, par un ensemble comportant seulement un demi avant-distributeur supérieur amovible fixé sur un demi anneau de scellement supérieur. A la partie inférieure, le flasque extérieur du distributeur est fixé directement sur un demi anneau de scellement.

- Simplification des circuits de graissage et de réfrigération des paliers et butées.

- Suppression du joint à air amont.

Caderousse:

- Mise au point de la solution chambre d'eau qui permet, outre une simplification des formes des ouvrages de

(') Le lecteur pourra se reporter à l'article de MM. J. Chevalied et H. Grraud, paru dans le numéro spécial de La Honille Blanche (No 2-3 de 1968: Compte rendu de la session des 16 et 17 novembre 1967 de la Société Hrydrotechnique de France). 
génie civil, le démontage de l'alternateur sans intervention notable sur les parties actives de la turbine.

b) Mise au point d'un type de groupe bulbe simplifié, dit «groupe fixe», prévu pour les centrales de Sauveterre et de Caderousse. Cette dernière centrale, qui comporte un équipement mixte, va nous permettre de faire le point actuel de l'évolution des conceptions de gros groupes bulbes, fixes et mobiles.

Les caractéristiques hydrauliques principales des groupes de Caderousse, de type bulbe amont en chambre d'eau, sont les suivantes:

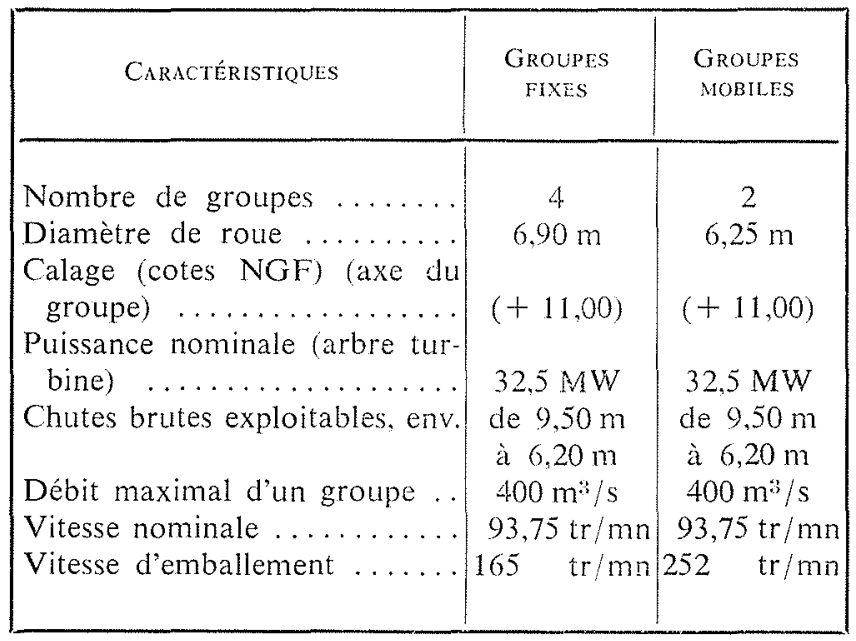

\subsection{Conception mécanique des groupes fixes ef mobiles.}

Du fait de leur implantation en chambre deau, les modifications suivantes ont été apportées aux groupes de Caderousse, par rapport à la famille Beaucaire-Gervans- $A_{2}$ vignon.

\subsubsection{Appuis DU BULBE.}

Les appuis du bulbe sont constitués:

- Pour les groupes fixes, par un distributeur en quatre parties et, pour les groupes réglables, par un avantdistributeur en deux parties, ces pièces étant directement scellées dans le béton primaire. L'anneau de scellement amont est ainsi supprimé.

Du point de vue mécanique, la fonction essentielle du distributeur, ou de l'avant-distributeur, est la reprise des efforts dûs au porte à faux du moulinet et des efforts d'origine hydraulique appliqués au distributeur et à la roue (couple et poussée). La transmission de ces efforts aux bétons primaires a fait l'objet d'une étude détaillée, en collaboration avec les ingénieurs de génie civil, et a permis d'aboutir à une solution présentant toute sécurité pour ces pièces essentielles à la tenue des machines. Une attention toute particulière a été apportée au dimensionnement de ces pièces mécano-soudées qui ne doivent permettre qu'une flèche très réduite au droit du palier turbine, compte tenu des faibles entrefers adoptés pour la roue turbine et le rotor alternateur.

- Par le croisillon amont supporté par deux pieds verticaux. L'ensemble croisillon-pieds verticaux assure la transmission au béton de la plus grande partie du couple hydraulique. La liaison croisillon-pieds verticaux amont a été conçue pour permettre un certain glissement axial relatif entre pieds et croisillon, afin de réduire les efforts axiaux d'origine thermique.

Bien que la rigidité de l'appui amont soit légèrement inférieure à celle de l'ensemble ogive-bras horizontaux et verticaux des groupes en conduit, la présence d'un distributeur complet, présentant une grande rigidité, a permis la suppression des bras horizontaux sans altération de la rigidité globale du groupe.

Cette suppression permet de simplifier considérablement l'ogive amont et de réduire le coût de la turbine, au prix d'une faible diminution des facilités d'accès à l'amont du bulbe. Cette sujétion a été facilement admise par les exploitants.

\subsubsection{ACCÈs a L'TNTÉrieur dU BUlbe.}

Ces accès sont évidemment conditionnés par la conception des appuis. Pour les groupes fixes comme pour les groupes mobiles, on dispose:

- à l'aval, d'avant-directrices creuses, permettant la soríe des barres de l'alternateur (avant-directrice supérieure), l'accès au palier turbine et au joint charbon à partir de la cosse turbine et le passage des tuyauteries;

- à l'amont, deux pieds verticaux qui permettent: l'un l'accès du personnel à la partie amont, l'autre le passage des tuyauteries.

\subsubsection{Montage et démontage.}

Du point de vue montage et démontage, les groupes en chambre d'eau présentent l'avantage, sur les groupes en conduit, de rendre les pièces fixes amont et lalternateur indépendants des parties actives de la turbine. De ce fait :

- au montage, après réglage et scellement du distributeur (ou de l'avant-distributeur), il est possible de monter simultanément les parties amont et aval de la machine; - s'il s'avérait nécessaire de procéder à un démontage complet de l'alternateur en cours d'exploitation, ce démontage est possible, sans aucune autre intervention sur la turbine proprement dite, que le calage du moulinet.

\section{2. - Groupes mobiles}

Mises à part les dispositions générales présentées ci-avant, ces groupes sont de conception classique et la coupe du groupe est représentée sur la figure 9.

On retrouve les dispositions de la ligne d'arbre à deux paliers: un palier turbine calculé pour une charge de $127 \mathrm{t}$, un palier amont chargé avec $63 \mathrm{t}$. La poussée hydraulique, do $290 \mathrm{t}$ sur la roue, est transmise à la butée à membranes située entre le palier amont et les alvéoles. Une contre. butée, de $590 \mathrm{t}$ de charge nominale, transmet, au croisillon support, la contre-poussée axiale lors des régimes transitoires.

Les parties fixes du bulbe sont analogues à celles des groupes en conduit du Rhône, à l'exception:

- - de l'ogive qui se réduit à un simple fond;

- de l'avant-distributeur, constitué d'une grille d'aubes complète;

- de l'anneau de scellement amont, qui est supprimé. 
Distributeur, roue, joints hydraulique et à air comprimé, paliers, butée, régulation, circuits de graissage et de réfrigération sont identiques aux ensembles correspondants de la famille Beaucaire-Gervans-Avignon.

\section{3. - Groupes fixes}

\subsection{Disposition mécanique d'ensemble.}

La disposition mécanique d'ensemble des groupes fixes est représentée sur la figure 10.

La conception de la ligne d'arbre est analogue à celle des groupes mobiles. Les alvéoles de distribution et le servomoteur de commande des pales sont évidemment supprimés, ce qui simplifie considérablement l'arbre turbine et permet de placer l'ensemble butée-contre-butée entre l'alternateur et la roue turbine. Les poussées hydrauliques sont ainsi transmises directement à l'avant-distributeur et n'intéressent plus la carcasse de l'alternateur. Cette disposition permet également de simplifier le croisillon amont.

Les parties fixes du bulbe comportent, d'amont en aval :

\subsubsection{A L'INTÉrieur du CONDUIT:}

- l'ogive fermant le groupe;

- le croisillon support du palier amont, le croisillon étant solidaire des deux pieds inférieurs scellés dans le béton du conduit;

- les pieds amont, qui sont aménagés pour permettre l'accès à la partie amont du groupe, le stockage des huiles de graissage et le passage des tuyauteries;

- la carcasse du stator de l'alternateur;

- l'anneau intérieur de l'avant-distributeur, support de butée et contre-butée;

- le cône de raccordement à la roue, supportant le palier turbine, et le joint d'étanchéité sur l'arbre, isolant l'intérieur du bulbe de l'eau du conduit.

\subsection{2. - A L'EXTÉrieur dU CONDUIT:}

- l'anneau extérieur de l'avant-distributeur, ancré dans le béton;

- la ceinture de roue, simplifiée par rapport aux bulbes classiques par l'adoption d'un profil cylindrique; la demi ceinture inférieure est scellée dans le béton;

- un cône aval, assurant le raccordement de la ceinture de roue avec le blindage de l'aspirateur scellé dans le béton.

Pour la demi-ceinture supérieure, un joint coulissant permet la libre dilatation de l'enveloppe extérieure du conduit dans la fosse turbine.

\subsection{Particularités technologiques.}

\subsubsection{ROUE.}

C'est une roue du type Kaplan, d'un diamètre extérieur de $6,900 \mathrm{~m}$, comportant les pales fixes rapportées sur le moyeu, d'un poids total de $58,6 \mathrm{t}\left(^{(g}\right)$. La coupe de la roue est représentée sur la figure 11.

$\left.{ }^{g}\right)$ Contre $65 \mathrm{t}$ pour les roues de groupes « réglables », de diamètre inférieur $(6,25 \mathrm{~m})$.
Cette coupe met en évidence les simplifications considérables apportées par cette solution: l'accouplement est réalisé par un goujon central unique et dix broches ajustées, le plateau d'accouplement de l'arbre étant supprimé. Les pales de la roue sont coulées en acier inoxydable à $13 \%$ de chrome et le moyeu est réalisé en acier faiblement allié.

\subsubsection{Paliers et butées.}

Les caractéristiques nominales de ces organes sont les suivantes :

- palier turbine ................... $110 \mathrm{t}$

- palier alternateur .................. $66 \mathrm{t}$

- butée .......................... $370 t$

- contre butée ..................... $560 t$

Le coussinet du palier turbine est réalisé en quatre parties, a fin de permettre son démontage par l'amont à travers le rotor de l'alternateur.

Les butée et contre-butée sont du type à patins indépendants montés sur membranes avec répartition hydrostatique des charges (type auto-compensé breveté).

Le grain mobile est constitué par le plateau d'accouplement de l'arbre turbine avec l'arbre alternateur.

Une pompe volumétrique, entrainée directement par l'arbre à l'amont du groupe, assure la circulation d'huile qui s'effectue, par gravité, à partir d'un bac supérieur.

\subsubsection{Distributeur.}

Le distributeur est un ensemble mécano-soudé, réalisé en deux éléments, et constitué d'un anneau extérieur, d'un anneau intérieur et de douze avant-directrices, dont huit pleines et quatre creuses.

L'anneau extérieur est ancré dans le béton primaire par l'intermédiaire de 150 tiges d'ancrage.

L'anneau intérieur assure la liaison entre la carcasse de l'alternateur et le cône turbine. Il constitue également le support de butée et contre-butée et la cloison d'étanchéité aval de l'enceinte alternateur.

Les directrices creuses, placées sur les axes verticaux et horizontaux, sont aménagées comme suit:

- verticale supérieure: sortie des barres de l'alternateur;

- verticale inférieure: accès au palier turbine et aux butées et passage des tuyauteries;

- horizontales : tuyauteries.

\section{4. - Particularités d'exploitation}

Toute régulation étant évidemment éliminée sur les groupes fixes, il est intéressant de donner ici quelques indications sur les conditions d'exploitation du groupe.

\subsection{Démarrage et arrêt.}

Comme il a déjà été indiqué, la mise en vitesse du groupe, le couplage en asynchrone et la prise de charge s'effectuent par levée progressive de la vanne aval.

Jusqu'à une vitesse inférieure à $60 \%$ de la vitesse nominale $\left(n_{0}\right)$, le graissage est assuré par un groupe moto-pompe 


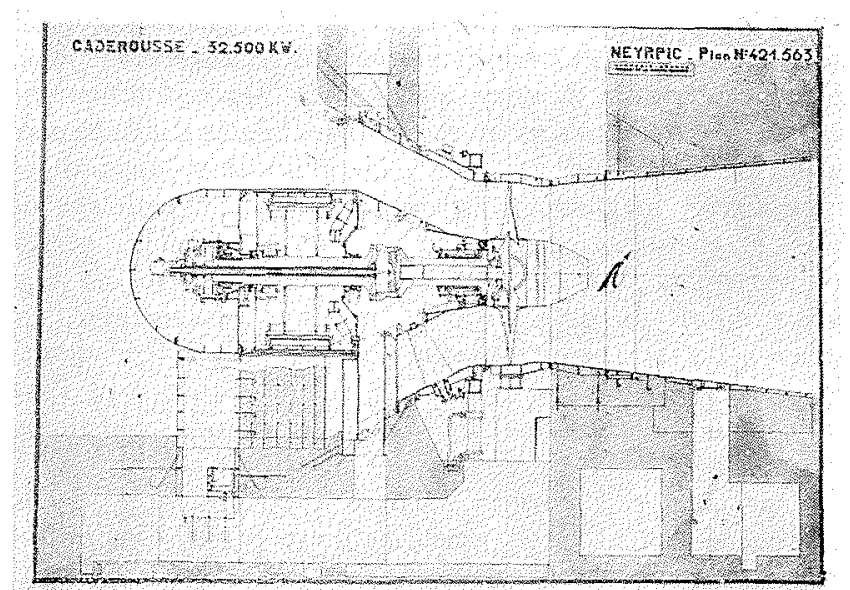

9/

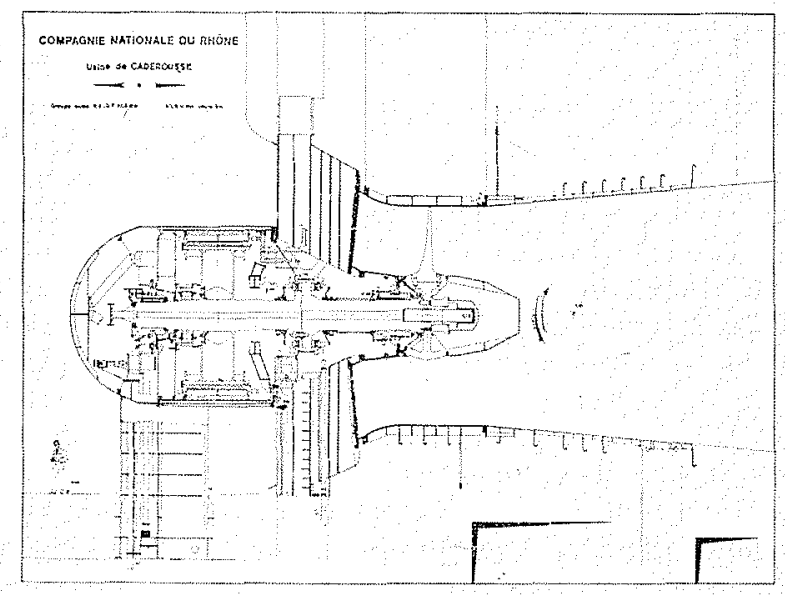

$10 /$

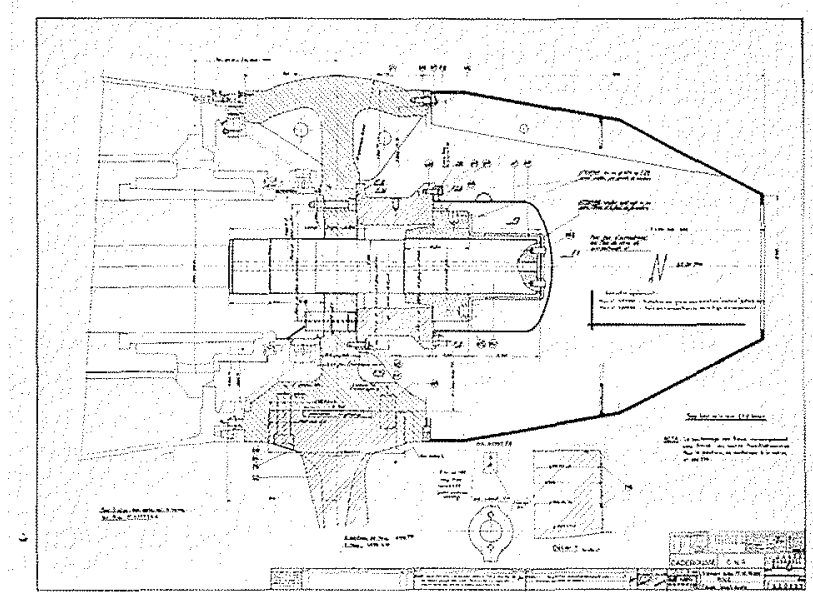

$11 /$ d'injection pour les paliers et un groupe moto-pompe extérieur pour l'ensemble des circuits d'huile. Lorsque la vitesse du groupe atteint $60 \%$ de la vitesse nominale, un relais provoque l'arrêt de ces deux pompes et la pompe, entraînée directement en bout d'arbre amont, assure seule le graissage.

Les arrêts volontaires, ou commandés par l'action d'une sécurité mécanique, sont assurés par fermeture de la vanne aval. Un contact de position, placé sur cette vanne, provoque l'ouverture du disjoncteur lorsque la puissance s'annule.

L'arrèt d'urgence, en cas de survitesse ou sous l'action d'une sécurité, provoque simultanément l'ordre de fermeture de la vanne aval et la disjonction du groupe.

\subsection{Marche en déchargeur.}

L'ordre de fonctionnement en déchargeur est donné par un contact détectant l'ouverture du disjoncteur de groupe.

Il est verrouillé par le contact «groupe en défaut».

Cet ordre de départ en déchargeur provoque:

- la désexcitation du groupe;

- la fermeture rapide de la vanne aval, qui se poursuit en fermeture lente dès que la vanne atteint une position déterminée ou que la vitesse du groupe décroît audessous de $85 \%$ de $n_{0}$.

La vanne aval, de nouveau ralentie à $77 \% n_{0}$, est stoppée lorsque la vitesse du groupe atteint environ $72 \%$ de $n_{o}$.

$\mathrm{Si}$, par suite de fuites, une descente lente de la vanne provoque une réduction de vitesse du groupe jusqu'à $67 \%$ de $n_{0}$, un ordre d'ouverture est donnée à la vanne qui est stoppée lorsque la vitesse du groupe atteint à nouveau $72 \%$ de $n_{o}$.

Le passage de la marche en déchargeur à la marche en turbine s'effectue par ouverture de la vanne aval et couplage en asynchrone.

\subsection{Sécurités contre l'emballement.}

La vitesse maximale d'emballement des groupes fixes $(165 \mathrm{tr} / \mathrm{mn})$ est notablement inférieure à celle des groupes mobiles, toutes choses égales par ailleurs, ce qui présente une sécurité en soi. De plus, en ce qui concerne la turbine elle-même, la structure interne du moyeu, avec les pales fixes, est beaucoup mieux adaptée à la transmission des efforts d'origine mécaniques et hydraulique que celle des moyeux à pales mobiles.

Le premier stade de la sécurité contre l'emballement est assuré par la fermeture de la vanne aval, sous l'action du détecteur de survitesse. Le deuxième stade de la protection est constitué par la vanne amont banalisée, capable d'assurer la coupure du plein débit d'emballement. Compte tenu du temps de mise en place de cette vanne, les groupes fixes sont calculés et garantis pour un fonctionnement continu de six heures à l'emballement.

\subsection{Montage.}

Les phases principales de montage des groupes fixes en chambre d'eau sont les suivantes:

- mise en place, réglage et bétonnage du blindage de l'aspirateur;

- mise en place et réglage du distributeur; bétonnage du mur aval de la chambre d'eau; 
- stockage provisoire des pieds amont et de l'anneau de scellement aval dans leurs logements;

- mise en place de l'ensemble alternateur, croisillon et ogive;

- liaisons pieds croisillons;

- assemblage alternateur-distributeur;

- bétonnage des pieds amont;

- parallèlement, mise en place et réglage de la demiceinture inférieure;

- liaison ceinture-distributeur;

- réglage de l'anneau de scellement aval et boulonnage avec la ceinture;

- dépose du moulinet dans la ceinture;

- accouplement turbine alternateur et réglage de la ligne d'arbre;

- montage de la demi-ceinture supérieure;

- bétonnage de la demi-ceinture inférieure et de l'anneau de scellement aval.

\section{5. - Conception des alternateurs}

\subsection{Dispositions générales et caractéristiques des alier- nateurs.}

Les alternateurs bulbes de Caderousse sont de conception très voisine de ceux des centrales antérieures (Beaucaire, Gervans, Avignon, Sauveterre) qui diffèrent eux-mêmes très peu entre eux.

En effet, bien que ces alternateurs (31,5 MVA, $\cos \Phi=0,98,93,75 \mathrm{tr} / \mathrm{mn}$ ) soient d'une puissance nominale inférieure à celle des alternateurs de Beaucaire $(35 \mathrm{MVA}$, $\cos \Phi=1,93,75 \mathrm{tr} / \mathrm{mnn}$ ), leurs parties actives ont été conservées et ont le même dimensionnement; ceci se justifie, non seulement par des raisons économiques de fabrication et de rechange, mais aussi par la facilité d'adaptation du système de refroidissement dans l'air comprimé à une puissance moindre.

C'est ainsi que la pression d'air de 3 bars, utilisée ì Beaucaire, a été conservée de façon à permettre aux alternateurs de fournir à pleine charge, et sur demande expresse du réseau, une certaine puissance réactive tout en conservant au rotor l'échauffement garanti; ainsi, ces groupes seront susceptibles de fournir chacun une puissance réactive d'un peu plus de 6 MVAR à la pleine charge active.

De même, la tension de $4900 \mathrm{~V}$ a été conservée et la diminution de la densité de courant dans l'enroulement statorique, qui en résulte, conduit à une réduction des pertes Joule du stator.

\subsection{Technologie de construction des alternateurs.}

\subsubsection{STATOR.}

La carcasse, insérée entre l'avant distributeur et le croisillon amont, est formée, comme pour les alternateurs antérieurs, par une structure en caissons. Elle est constituée par un assemblage de viroles minces et de poutres d'entretoisement. La place, ainsi ménagée, permet de loger les échangeurs de chaleur constitués par des panneaux de tôles minces munis d'ailettes en cuivre, panneaux qui ferment les caissons de la carcasse du côté extérieur.

Afin de faciliter l'évacuation des calories vers l'eau, tout en assurant une bonne protection contre la corrosion, la carcasse est enduite, extérieurement, d'un revêtement protecteur à base de zinc, ce qui permet, par rapport à une peinture classique, un gain de plusieurs degrés sur la température de l'air froid.

Le circuit magnétique est constitué par un empilage de tôles magnétiques, d'épaisseur $0,35 \mathrm{~mm}$, à faibles pertes, soigneusement ébavurées et isolées, serrées entre des segments munis de doigts soudés en acier amagnétique. La pression de serrage est obtenue par des tiges indépendantes des barreaux clavettes. Une fente étroite, située dans l'axe de chaque dent, assure une ventilation longitudinale et permet une bonne évacuation des pertes du stator.

L'enroulement statorique à barres Roebel est isolé suivant le procédé Isoténax: les jonctions entre barres sont isolées par des capots remplis de résine.

La sortie des phases, à l'extérieur du bulbe, est assurée, à l'aval, à travers une avant-directrice verticale, au lieu d'un bras vertical disposé à l'amont (Beaucaire, Gervans ou Avignon), ou d'une avant-directrice horizontale, disposée à l'aval, à Sauveterre. Le point neutre est formé à l'intérieur de l'alternateur.

\subsubsection{ROTOR.}

Le centre rotor est réalisé par un arbre creux, en acier forgé, sur lequel est soudé un croisillon, celui-ci étant enveloppé par une jante, en tôle épaisse, roulée et soudée. La vitesse d'emballement plus faible des groupes fixes a permis d'alléger le croisillon et la jante, qui sont alors dimensionnés, non plus par les contraintes mécaniques, mais par le passage du flux de pôle à pồle $\left(^{h}\right)$. L'allègement du croisillon et de la jante se traduit par une économie de l'ordre de $0,8 \%$ du prix de l'alternateur.

Le forage intérieur de l'arbre creux des groupes fixes sert uniquement au contrôle de la qualité de la matière, alors que l'arbre creux des groupes mobiles est utilisé pour ce même contrôle, mais aussi pour le passage des canalisations d'huile de commande des pales de la roue.

Les noyaux polaires sont réalisés par un empilage de tôles magnétiques minces isolées (épaisseur $0,35 \mathrm{~mm}$ ), afin d'éloigner les possibilités du phénomène d'électro-érosion des barres amortisseurs.

Les bobines de chaque barre occupent toute la fenêtre interpolaire et sont superposées, ce qui permet d'annuler les composantes tangentielles de la force centrifuge et d'éliminer le calage latéral des bobines, incompatible avec une ventilation axiale.

\subsubsection{Ventilation.}

La ventilation axiale est la plus logique dans le cas des groupes bulbes, où la vitesse périphérique est trop faible pour pouvoir utiliser la ventilation radiale par le rotor. La circulation de l'air est donc assurée par deux groupes motoventilateurs situés à l'amont de l'alternateur.

Mais, étant donnée la grande longueur de la machine, et en vue de réduire les pertes de charge dues au passage de

(h) Les rotors des groupes mobiles n'ont, par contre, pas été allégés. 
l'air de refroidissement, la ventilation a été scindée en deux parties: au milieu de la machine l'air chaud, provenant du refroidissement de la partie aval, est extrait par des évents dans le circuit magnétique, et de l'air froid est alors réintroduit, par des trous dans la jante, afin de refroidir la partie amont. Les deux circuits d'air chaud sont repris par les moto-ventilateurs situés à l'amont et envoyés dans l'échangeur aileté de la carcasse.

Les moteurs des ventilateurs placés dans la calotte de fermeture du bulbe, où règne la pression 3 bars, sont classiques et non refroidis par eau: l'air de ventilation est refroidi par léchage sur la paroi de la calotte. Les moteurs alimentés par les auxiliaires, branchés en soutirage direct aux bornes de l'alternateur, auront, pour les groupes mobiles, à supporter les survitesses en cas de déclenchement à pleine charge et les sous-vitesses en cas de marche en déchargeur. Par contre, pour les groupes fixes et dans les deux cas évoqués ci-avant, l'alternateur sera désexcité et les moteurs seront isolés des auxiliaires.

\subsubsection{FrEINAGE ET BLOCAGE DU ROTOR.}

Les groupes fixes, comme à Sauveterre, ne possèdent pas de système de freinage, par contre les groupes mobiles, comme à Avignon, en possèdent un, par action de vérins à air comprimé sur une jante de freinage située à l'aval du rotor.

Le dispositif de blocage du rotor à l'arrêt et de son virage a été reporté du côté de l'accès, soit à l'amont.

\subsection{Excitation.}

Tous les alternateurs sont excités par un système d'excitation comportant un alternateur inversé (excitateur) et un ensemble de diodes tournantes fixées sur les bras du rotor. L'excitation de cet alternateur est fournie par un transformateur auxiliaire branché aux bornes de la machine.

Cette solution supprime les sujétions d'exploitation qu'imposaient les balais dans les premiers groupes bulbes.

La conception électro-magnétique des alternateurs de Caderousse étant identique à celle des machines des centrales antérieures, il est normal de trouver des caractéristiques d'excitation semblables.

En ce qui concerne le dispositif de limitation des surtensions rotoriques, qui prennent naissance lors des couplages asynchrones, une certaine évolution a été introduite depuis les alternateurs de Beaucaire: celix-ci sont munis d'une résistance linéaire, placée en parallèle aux bornes du rotor, et mise en service par des thyristors. Ces thyristors sont allumés par l'apparition même de la surtension et sont éteints par le fonctionnement normal de l'excitateur.

Les alternateurs de Caderousse, comme ceux de Gervans, Avignon et Sauveterre, sont équipés d'une résistance non linéaire raccordée, en permanence, aux bornes du circuit inducteur. Cette résistance est constituée de seize ensembles de douze éléments (type CARBOHM) tous (ensembles et éléments) insérés en parallèle. Chaque élément est capable d'absorber $45 \mathrm{~mA}$ sous $330 \mathrm{~V}$ et $6 \mathrm{~A}$ sous $900 \mathrm{~V}$.

Les couplages sôu pleine tension à $5 \%$ de glissement correspondent à peu près au régime $6 \mathrm{~A}, 900 \mathrm{~V}$, et le régime nominal à moins de $45 \mathrm{~mA}$.

\section{CONCLUSIONS ET PERSPECTIVES}

En accord avec les constructeurs des turbines et des alternateurs, qui ont la responsabilité de la conception et de la construction des machines, nous avons présenté les problèmes et les choix qui se posent aux maître d'œuvre et exploitants pour l'incorporation, dans une même usine, de deux types différents de groupes bulbes.

Par rapport aux centrales précédentes, Pierre-Bénite, Vallabrègues, Gervans et Avignon, toute la conception de la centrale de Caderousse a été repensée : les entrées en chambre d'eau ont été substituées aux entrées en conduit, afin de recevoir la vanne banalisée amont, l'incorporation des matériels dans les maçonneries (l'avant distributeur pour les groupes réglables et distributeur pour les groupes fixes), les circulations et la desserte de la centrale, les vannes aval standardisées.

Tous ces problèmes se traduisent, en définitive, par une suite de compromis et de décisions que nous avons cherché à faire ressortir.

De mème, les hydrauliciens et les constructeurs turbinier et électricien précisent les problèmes auxquels ils ont été confrontés pour livrer un matériel fiable, capable de donner entière satisfaction aux maître d'œuvre et exploitants.

Le cas de l'usine de Caderousse, qui comporte un équipement mixte, avec des groupes réglables et des groupes fixes, constitue un cas particulier; la solution qui a été retenue nétant pas automatiquement valable pour un autre aménagement.

Dans un autre site, les groupes fixes peuvent ne pas présenter le même intérêt, par suite de la variation importante des débits classés ou des hauteurs de chute classées, ou même de ces deux facteurs à la fois, et peuvent conduire: soit à une perte trop grande d'énergie, incompatible avec l'économie d'investissement, soit à un surdimensionnement des alternateurs. Par contre, d'autres sites, où la chute reste très constante, peuvent être plus favorables que Caderousse et inciter à un équipement complet en groupes simplifiés. L'équipement «mixte » de Caderousse, qui permet d'abaisser notablement le coût de la centrale, est un nouveau pas vers la rentabilisation des usines de basses chutes.

Les groupes fixes, en abaissant la charge sur le palier turbine, permettent de reculer les limites constructives des groupes à deux paliers. Pour ces groupes fixes, on peut envisager, dès maintenant, un diamètre de roue voisin de $8 \mathrm{~m}$, alors que pour les groupes mobiles, nous avons jugé sage, quant à nous, de ne pas dépasser des cliamètres de $6,50 \mathrm{~m}$. Il appartient aux constructeurs, en fonction de leurs études les plus récentes, de dire ce que vaut actuellement cette limite. 


\title{
DISCUSSION DES COMMUNICATIONS
}

\author{
M. J. COTILlon : \\ LES GROUPES BULBES. DE RÖTIN EN AVIGNON : L'ESSOR D’UNE TECHNIQUE. \\ M. BESLIN : \\ IDÉES ACTUELLES SUR I.A CONCEPTION DES GROUPES BULBES \\ EXEMPLE DE CADEROUSSE
}

Président: M. P. CAZENAVE

M. DUPort (SOGREAH, Division des Techniques Industrielles) présente les trois remarques ci-après :

$1^{\circ}$ Les avantages «hydrauliques» des groupes bulbes (vitesses et puissances spécifiques élevées) n'étaient pas connus - en tout cas pas acquis - à l'époque à laquelle se réfère $M$. Cotillon ( 1 de son mémoire). C'est au cours de la mise au point de l'équipement de la Rance que ces avantages nous sont apparus complètement. Cela contribue à expliquer que la technique en question ne se soit pas imposée plus tôt. 11 n'en reste pas moins que la raison essentielle de ce fait - comme l'indique M. Cótullon - réside dans l'absence, à l'époque, d'alternateurs adaptés.

$2^{\circ}$ La non-prise en considération des groupes-puits pour l'étude de la Rance résulte moins des incertitudes de cette technique que du fait que l'économic d'investissement qu'elle aurait apportée, était jugée insuffisante pour justifier la «mutation» qu'elle représentait. La préoccupation essentielle, en 1951, était, en effet, celle de la réduction du coût d'investissement; c'est cette préoccupation qui a motivé l'option en faveur des groupes bulbes. Immédiatement après, on vérifia la bonne aptitude des groupes bulbes aux fonctionnements «inverses» en pompe et en turbine. Parallèlement, l'intérêt économique d'une production modulée sur la «demande» était analysée, et il fut ainsi décidé de tirer pleinement parti des cycles marémoteurs que permet l'utilisation des groupes bulbes.

$3^{\circ}$ (Cf. \$ 5.4). La rédaction très concise du troisième alinéa ne risque-t-elle pas de prêter à confusion? Le Verbois et Bonneville sont des expériences de coupure amont qui ont montré «indirectement » - par les difficultés du bâtardage amont - l'intérêt essenticl du bâtardage aval. Seysscl, en 1951, a été la première expérience de bâtardage aval, dont le plein succès a été pour une grande part dans l'adoption du procédé.

M. Lefoulon (ancien directeur de la Région d'Equipement nord de l'E.D.F.) fait ensuite une intéressante intervention quil a bien voulu résumer en ces termes:

En complément de l'historique très intéressant de M. Cotrulon, je veux donner quelques précisions en ce qui concerne l'aménagement du Rhin. En 1956, un accord franco-allemand confirme à la France la mission et la maîtrise d'œuvre de l'aménagement du Rhin à l'aval de Vogelgrïn jusqu'à Strasbourg. Fin 1956, le projet d'équipement de ce tronçon du fleuve pour un débit de $1400 \mathrm{~m}^{3} / \mathrm{s}$ comprend à l'amont deux biefs de même hauteur de chute, $12,50 \mathrm{~m}$ environ: Marckolsheim et Rhinau et à l'aval deux biefs de même hauteur de chute, $10,60 \mathrm{~m}$ à $11 \mathrm{~m}$ : Gerstheim et Strasbourg. Ce découpage permet d'avoir deux ensembles d'usines jumelles, équipés chacun avec les mêmes groupes avec les économies qui en découlent. Fin 1955 , l'usine de Marckolsheim est conçue avec quatre groupes verticaux Kaplan classiques de $350 \mathrm{~m}^{3} / \mathrm{s}$. Lorsqu'en 1958 , il s'agit de décider de l'équipement de l'usine de Rhinau, les études sur les groupes bulbes et la réalisation d'un prototype d'environ $10 \mathrm{MW}$ à Saint-Malo nous incitent à demander aux constructeurs le projet de groupes de puissance maximum pour une chute de $12,50 \mathrm{~m}$. A cette date, ils ne peuvent nous garantil qu'un groupe de $17 \mathrm{MW}$ de puissance, lequel conduit à un projet de Rhinau à huit groupes. Avec ce nombre de groupes, il n'y a pas d'économie de génie civil sur la solution à quatre groupes verticaux classiques, et compte tenu de la situation de l'usine sur la frontière, sous le regard de nos voisins d'Outre-Rhin, la prudence est de reconduire à Rhinau les groupes de Marckolsheim comme prévu en 1956.
J'estime, en effet, que les difficultés de la construction des groupes bulbes à axe horizontal résident plus dans la réalisation de l'alternateur de grande puissance immergé et accouplé directement à la turbine qu'à la turbine même, laquelle fonctionne à l'inverse des hélices des bateaux en service depuis longtemps.

En 1960, les études de l'usine de Gerstheim, de $10,60 \mathrm{~m}$ de hauteur de chutc, sont entreprises. Deux ans se sont écoulés et le groupe de Saint-Malo défnitivement au point après de nombreux essais permet de fixer les caractéristiques des groupes qui équiperont l'usine de la Rance. Les constructeurs consultés pour Gerstheim nous donnent lassurance de pouvoir construire, sans extrapolation trop hardie, un groupe de $23 \mathrm{MW}$ qui permet de concevoir l'usine avec six groupes. Aucune hésitation n'est plus permise, les économies sur le génie civil et sur le nombre des déchargeurs conduisent à opter pour les groupes bulbes fin 1960. Les deux usines de Gerstheim et de Strasbourg seront équipées de douze groupes semblables de $23 \mathrm{MW}$ de puissance unitaire.

Permettcz-moi dajouter une remarque très actuelle. Maintenant que les groupes bulbes sont parfaitement au point et assurent l'aménagement des basses chutes avec le maximum d'économies, il apparaît pour le moins paradoxal qu'on réalise l'équipement du Rhin à l'aval de Strasbourg-Gambsheim avec un sous-équipement à quatre groupes absorbant $1000 \mathrm{~m}^{3 /} / \mathrm{s}$ alors que les usines d'amont peuvent turbiner $1400 \mathrm{~m}^{3} / \mathrm{s}$. Je suis loin d'être convaincu de l'intérêt économique, dans l'avenir, d'un tel sous-équipement. Je crois que l'installation d'un cinquième groupe, d'une part apporterait à un coût marginal, une production annuelle de 50 à 100 millions de $\mathrm{kWh}$ suivant que les quatre groupes absorberaient au maximum 1000 on $1100 \mathrm{~m}^{3}$. d'autre part, elle «assurerait» la production escomptée des quatre groupes. Je doute, en effet, quion puisse demander à des groupes et à leurs accessoires, des fonctionnements de plus de $8000 \mathrm{~h}$ par an au fil des années.

M. Petermann (Ateliers des Charmilles) complète l'historique de M. Corillon par les informations ci-après qui concrétisent les possibilités des groupes bulbes dans l'avenir immédiat:

Il s'agit de l'usine d'Ottensheim en construction sur le Danube et de l'usine de Altenworth sur le même fleuve dont les machines sont en cours d'essais sur modèle réduit. Voici les caractéristiques essentielles de ces groupes:

a) Ottensheim :

- diamètre de la roue .... $5,6 \mathrm{~m}$;

- hauteur de chute ...... $12 \mathrm{~m}$

- puissance unitaire ..... $25 \mathrm{MW}$

- nombre de groupes .... 6.

b) Altenworth:

- diamètre de la rone ... $6,1 \mathrm{~m}$

- hauteur de chute ...... $15 \mathrm{~m}$

- puissance unitaire ..... $40 \mathrm{MW}$

- nombre de groupes .... 9.

Sur modèle, le rendement maximum des turbines est de 93,4\%. L'utilisation optimale des groupes conduit à un rendement global de l'usine supérieur à $92 \%$ pendant $72 \%$ de l'année.

Se référant au point 1 de l'intervention de M. DUPORT, M. MEysTRE (Escher Wyss) donne les précisions suivantes:

Escher Wyss procéda déjà avant 1930 à des essais comparatifs sur modèles de turbines Kaplan de type bulbe selon les brevets dont M. Cotillon a bien voulu parler. Quelques résultats figurent dans 
un Bulletin Edcher Wyss de 1931. Nous ne songions évidemment pas encore aux grandes dimensions, néanmoins il est intéressant de relever que Röstin avait déjà un bulbe combiné avec le stator de l'alternateur; que celui-ci était en outre accouplé directement à la roue; enfin que le diamètre de la roue de $1350 \mathrm{~mm}$ faisait déjà $123 \%$ de celui du bulbe, soit un rapport aujourd'hui respecté.

Uniquement les ennuis du côté du refroidissement de la machine électrique condamnèrent momentanément cette construction. Le manque de confiance qui en résulta en l'avenir immédiat des groupes bulbes et, de ce fait, le manque de crédits pour la recherche dans cette direction chez les électriciens freina l'évolution vers une construction meilleure.

Comme réalisation actuelle de grande dimension, notre collègue M. Petermann vient de vous citer les groupes à l'étude de $6,10 \mathrm{~m}$ de diamètre; vous savez sans doute que sont actuellement à l'étude définitive, en collaboration entre la France et Escher Wyss, les groupes d'IfTezheim, de $5,80 \mathrm{~m}$ de diamètre et de $29 \mathrm{MW}$, qui feront partie de l'équipement hydroélectrique du Rhin, à l'aval de Strasbourg.

Sur l'invitation de $M$. le Président, M. Cotillon répond, comme suit, aux interventions qui précèdent:

Je voudrais profiter de la présence de M. MEYSTRE pour remercier Escher-Wyss d'avoir bien voulu procéder à des recherches de brevets fort anciens, ce qui me donne le plaisir de disposer aujourd'hui de trois brevets dont peu de gens, sans doute, avaient entendu parler.

Je suis vraiment surpris de la modération de M. LEFoulon en ce qui concerne l'équipement à l'aval de Strasbourg; je pense, quant à moi, que c'est six groupes qu'il aurait fallu installer.

A M. Petermann, des Charmilles, je dirai que je n'ai pas parlé des projets parce que c'est un domaine dont traitera M. CAsAccr. Mais lorsque les communications de cette Session seront publiées, il y aura, en annexe, une liste des groupes bulbes en fonctionnement, en construction et en projet et je serais très reconnaissant à $M$. PETERMANN de nous fournir les renseignements qu'il aurait sur des groupes qui pourraient être à l'étude chez des constructeurs suisses. Je fais la même demande à Escher-Wyss, afin que notre liste de groupes bulbes soit aussi complète que possible.

A M. Duport, j'ai trois choses à dire, puisqu'il a parlé sur trois points.

M. Duport a eu le privilège de lire une version provisoire de mon texte. Elle est provisoire, parce que, quand on écrit l'histoire et qu'on dispose de peu de temps, de peu de pages, on est obligé de faire des raccourcis qui se révèlent ensuite trop hardis. Aussi, je suis bien d'accord avec vous: quand je parle de l'écoulement axial après coup, j'ai tendance à lui donner toutes les vertus qu'on lui a découvertes au fil des jours, ce qui conduit à s'étonner que, dès 1930, voyant que cet écoulement axial avait tant de vertus, les constructeurs ne se soient pas acharnés pour le faire triompher. 11 semble cependant, comme vient de le dire M. Meystre, que cela n'ait pas échappé à certains et que, si un délai assez long s'est écoulé, c'est que le client, qui est plus exploitant que maître d'œuvre d'un équipement, demande un certain temps pour se laisser convaincre.

En ce qui concerne le groupe-puits - et c'est une des raisons pour lesquelles j'avais dit que mon exposé était provisoire - la phrase, telle qu'elle est rédigée, semble condamner le groupe-puits; ce n'était pas ce que je voulais dire et c'est un des points sur lesquels mon texte provisoire scra rectifié.

En ce qui concerne les essais de vanne-aval, là aussi, ma phrase est mal tournée; elle peut laisser penser qu'aussi bien à Bonneville qu'à Verbois, on a fait des essais de vanne aval, alors que, manifestement, ni à Bonneville, ni à Verbois, il n'y a de vanne aval.

Je crois avoir répondu sur tous les points qui ont été soulevés et n'avoir rien oublié.

Cette dernière remarque, observe M. le Président, me concuit à souligner l'importance du travail d'équipe que les conférenciers de cette séance ont été conduits à faire en collectionnant un nombre considérable d'informations recueillies auprès de nombreuses personnes. Il est toujours extrêmement difficile, dans des cas comme ceuxlà, d'arriver à faire une œuvre de présentation originale faisant passer l'idée de l'auteur sans trahir l'idée des co-auteurs qui ont fourni des informations. Chacun de ceux qui ont fait des rapports ici a senti cette difficulté. ll y avait un très gros travail préliminaire. Les auteurs de communications ont tous tenu à dire que leur texte ćtait provisoire et qu'ils le mettraient définitivement au point après les interventions que nous aurions en séance.

Je vais maintenant passer la parole à $M$. André qui a le privilège de constituer un centre d'information des plus complets: c'est lui, en effet, qui reçoit toutes les informations concernant les difficultés rencontrées sur tous les groupes hydroélectriques exploités par Electricité de France. Nul ne pouvait être mieux placé que lui pour venir vous exposer le bilan de l'exploitation de toutes ces machines.

$$
\text { * }
$$

Quelques jours après la Session, M. René Langlors (contrôleur général honoraire, E.D.F.) — qui n’avait pu être présent au moment de la présentation du rapport de M. Cotillon - nous a adressé l'intéressante contribution écrite, ci-après, à la discussion de ce mémoire :

Parmi les avantages que présentent les groupes bulbes, M. Cotrllon fait état de leur faculté de fonctionnement en déchargeur, particulièrement intéressante dans les aménagements de basses chutes.

$1^{\circ}$ Je voudrais signaler qu'à vrai dire, elle me paraissait avoir un caractère potentiel dès l'origine, dans les groupes classiques à turbines Kaplan, đu fait de l'orientabilité de leurs pales.

C'est seulement bien plus tard que fut mise en évidence cette faculté, lors des essais de fonctionnement en déconjugaison des pales de la roue et du distributeur, signalés par M. Cotillon et effectués à partir de 1955 par la C.N.R. et E.D.F. respectivement sur le Rhône et la Garonne, avec des groupes Kaplan verticaux (1).

Ces essais firent alors apparaître un pouvoir de dsccharge indiqué dans le rapport, de l'ordre de

- $30 \%$ du débit nominal en vitesse hyposynchrone.

$2^{\circ}$ Mais, finalement, c'est la combinaison des deux éléments d'amélioration - d'ailleurs entièrement distincts et, également signalés par M. Cotrllon - à savoir :

- utilisation de vannes à coupure rapide à l'aval;

- et utilisation de groupes bulbes;

qui devrait se révéler déterminante, permettant ainsi de porter le pouvoir de décharge des gros bulbes de rivière récents au chiffre énoncé de :

$-70 \%$ du débit nominal, en vitesse hyposynchrone.

$3^{\circ}$ Afin de compléter ces informations, je pense intéressant de préciser que les essais que nous avons effectués en 1959, sur le groupe bulbe expérimental de rivière de Beaumont-Monteux, - muni d'une vanne de coupure amont, - avaient déjà permis d’obtenir un pouvoir de décharge atteignant sensiblement:

— de 50 à $55 \%$ du débit nominal, en vitesse synchrone ou légèrement hypersynchrone,

ce fonctionnement s'entendant pales ouvertes et sous une ouverture du distributeur de $5 / 10$.

On avait même atteint en fonctionnement transitoire, une décharge de :

$-75 \%$ du débit nominal.

Cette marche obtenue avec ouverture du distributeur ì $6 / 10$, avait conduit à une survitesse de $25 \%$; mais clle n'avait duré que quelques instants en raison des vibrations dues à un écoulement particulièrement turbulent dans la roue.

Un tel résultat préfigurait en quelque sorte, ce qui devait être obtenu plus tard, dans de meilleures conditions d'écoulement. »

(1) N.D.L.R. : Cf, No Spécial 2.3/1968 de La Fonille Blanche 


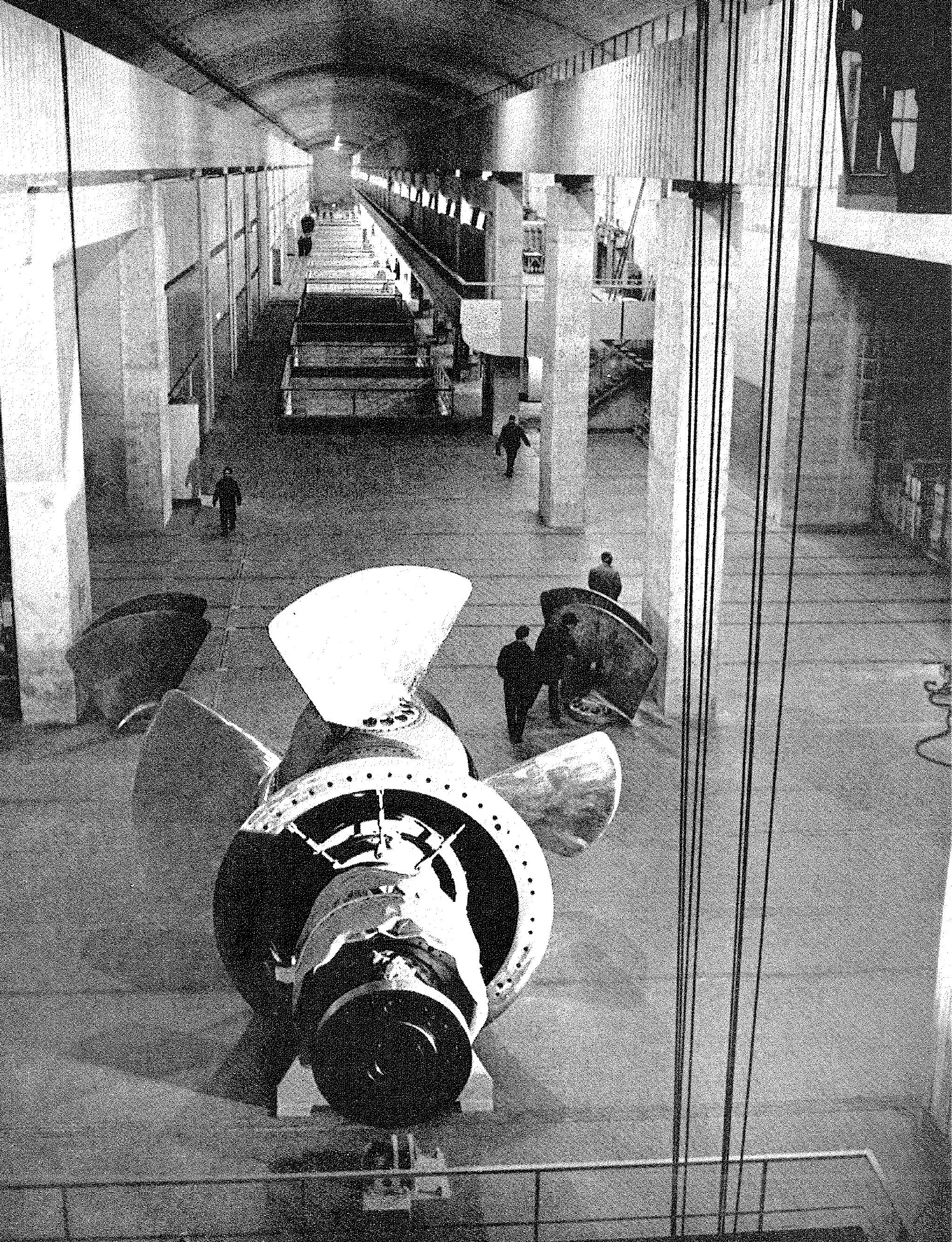

